\title{
Relation of the External Branch of the Superior Laryngeal Nerve to the Superior Pole of the Thyroid Gland: An Anatomical Study
}

\author{
D. NAIDOO,${ }^{1}$ J.M. BOON, ${ }^{1 \dagger}$ C.J. MIENY, ${ }^{2}$ P.J. BECKER, ${ }^{3}$ AND A.-N. VAN SCHOOR ${ }^{1 *}$ \\ ${ }^{1}$ Department of Anatomy, Section of Clinical Anatomy, Faculty of Health Sciences, School of Medicine, University of Pretoria, \\ Pretoria, South, Africa ${ }^{2}$ Department of Surgery, Faculty of Health Sciences, School of Medicine, University of Pretoria, Pretoria, \\ South Africa ${ }^{3}$ Unit of Biostatistics, Medical Research Council, South Africa \\ ${ }^{\dagger}$ Deceased
}

The external branch of the superior laryngeal nerve (ELN) is intimately associated with the superior thyroid artery (STA) in relation to the superior pole of the thyroid gland, rendering it vulnerable to injury during the ligation of this vessel during thyroidectomy. Although most texts acknowledge the fact that the nerve is in close relation to the STA, there has not been an anatomical study to relate the position of the ELN to the superior pole of the thyroid gland. The aim of this study was to determine the shortest distance, from the most superior point of the thyroid gland, to the ELN. Bilateral micro-dissection on 43 adult cadavers, excluding those with thyroid pathology and previous thyroidectomies, was undertaken. The most superior point of the superior pole of the thyroid gland was identified and the shortest distance to the ELN was measured with a digital calliper (accuracy $0.01 \mathrm{~mm}$ ). The metric study indicated a mean distance from the ELN to the superior pole of a normal sized thyroid gland of $5.76 \mathrm{~mm}$ (range: 2.00-11.26) on the right, and $6.17 \mathrm{~mm}$ (range: 2.78-13.48) on the left. From the literature, it is clear that the ELN may even be closer to the superior pole of an enlarged thyroid gland. The recommendation to stay on the substance of the superior pole of the thyroid gland when ligating the STA remains valid, as the nerve is extremely close in relation to the superior pole of the normal thyroid gland.

\section{INTRODUCTION}

Thyroidectomy is a common general surgical operation, which has enjoyed a relatively colourful history, advocated for the treatment of a wide variety of thyroid diseases, including Grave's disease (hyperthyroidism), Hashimoto's thyroiditis (hypothyroidism), multi-nodular goiter, and thyroid carcinoma (Anonymous, 1970).

The major concern in thyroid gland surgery is morbidity because of injury of anatomical structures (Wagner and Seiler, 1994; Moreau et al., 1998). Three important structures are necessary to preserve during thyroidectomy, namely the recurrent laryngeal nerve (RLN), the parathyroid glands, and the external branch of the superior laryngeal nerve (external laryngeal nerve or ELN) (Delbridge et al., 1992). The complication most feared by both the patient and surgeon is damage to the RLN (Wagner and Seiler, 1994). Thus, an enormous volume of work has been done to document the surgical anatomy of the RLN, to protect the nerve during surgery (Lekacos et al., 1987; Moreau et al., 1998; Hurtado-Lopez et al., 2005), all of which including the following parameters: origin of the RLN, number of terminal divisions of the RLN, relation of the RLN to the suspensory ligament of Berry, Zuckerkandl's tuberculum, and the inferior thyroid artery (ITA).

Over the years, the ELN has received much less attention and emphasis than the RLN during thyroid gland surgery (Durham and Harrison, 1964; Moosman and DeWeese, 1968; Droulias et al., 1976; Friedman and Toriumi, 1986; Lekacos et al., 1987). In fact, Lennquist et al. (1987) has described this nerve as the "neglected" nerve in thyroid 


\section{openUP}

gland surgery, despite the fact that injury to these nerves can also cause significant disability. Perhaps the most famous person who sustained damage to the ELN during thyroid gland surgery was Amelita Galli-Curci, the great coloratura soprano; postoperatively she discovered that her register had fallen to that of a lyric soprano. Her voice had become hoarse, and critics wrote, "The amazing voice is gone, perhaps forever. Instead of cream velvet jeweled with coloratura splendor there was an unsteady little lyric soprano quavering like a sad ghost pleading for reincarnation" (Chosky and Nicholson, 1996).

The superior laryngeal nerve (SLN) arises from the inferior ganglion of the tenth cranial nerve, near its exit from the jugular foramen of the skull (Berry et al., 1995). Within $2 \mathrm{~cm}$ of its origin, high up in the cervical region, the SLN divides into a larger internal branch and a smaller ELN (Durham and Harrison, 1964; Moosman and DeWeese, 1968). The ELN then descends to supply the inferior constrictor and cricothyroid muscles. The ELN is a small and somewhat flattened nerve; it is the only motor innervation of the cricothyroid muscle, an extra-laryngeal muscle that serves as a tensor of the vocal folds (Cernea et al., 1992a,b; Chosky and Nicholson, 1996).

Unilateral injury of the ELN may result in mild voice huskiness; however, bilateral injury frequently results in much more devastating consequences. Temporary or permanent paralysis of the cricothyroid muscle results in any one, or more, of the following changes in the quality of one's voice: hoarseness, weakness, huskiness, decreased range of pitch or volume, inability to produce certain sounds, and voice fatigue after extensive use (Moosman and DeWeese, 1968; Droulias et al., 1976; Friedman and Toriumi, 1986; Lekacos et al., 1987; Lennquist et al., 1987; Cernea et al., 1992a,b; Delbridge et al., 1992; Teitelbaum and Wening, 1995; Chosky and Nicholson, 1996; Hurtado-Lopez et al., 2005). In most individuals, these changes tend only to be a nuisance, but can prove to be catastrophic in those who depend on their voices professionally, such as singers, lawyers, lecturers, and public speakers.

Meticulous dissection is therefore imperative to avoid injury to the ELN, thus it is of utmost importance to have adequate knowledge of the anatomic variations of the course of the nerve. Although most texts acknowledge the fact that the nerve is in close relation to the superior thyroid artery (STA), there has not been to the best of our knowledge an anatomical study to relate the position of the ELN to the superior pole of the thyroid gland.

\section{AIMS}

This study aimed to determine the shortest distance from the most superior point of the superior pole of the thyroid gland to the ELN.

\section{MATERIALS AND METHODS}

A number of 43 adult cadavers were included in the study, exhibiting the following demographic profile: Sex: 32 males and 11 females; Age (years), mean: 59, median: 62, interquartile range (IR): 9; Height (cm), mean: 167.85, median: 167.64, IR: 2.54; Mass (kg), mean: 51.7, median: 167, IR: 4.35.

The cadavers were obtained from the Department of Anatomy, University of Pretoria, under the Human Tissues Act, Act 65 of 1983.

A total of 41 right and 43 left dissections were undertaken. None of the cadavers had previous thyroid surgery. All 
cadavers were fixed and placed in the anatomical supine position. The necks of the cadavers were placed in a semi-extended position, similar to the position in the operating room during a thyroidectomy.

The technique applied was that of bilateral micro-dissection. A vertical midline incision was made, extending from the suprasternal notch to the tip of the mandible. A curved horizontal incision was made along the clavicle on both sides, extending from the suprasternal notch to the acromion. As a result, two bilateral skin flaps were created and reflected superiorly. The platysma was then incised, and the layer of fascia covering the strap muscles was incised in the midline. Subsequently, the sternocleidomastoid, thyrohyoid, omohyoid, and sternohyoid muscles were exposed and loosened. The sternothyroid muscles were horizontally incised and carefully reflected superiorly, exposing the anterior surface of the superior pole of the thyroid gland. Meticulous dissection of the superior pole of the thyroid, superior thyroid vessels, and the ELN was undertaken.

Following identification of the above-mentioned structures, a marking pin was placed on the most superior point on the superior pole of the thyroid gland, at the point where the STA reaches the gland (Figs. 1 and 2). A digital calliper calibrated in $\mathrm{mm}$ (accuracy: $0.1 \mathrm{~mm}$ ) was then used to measure the shortest distance, from the tip of the needle at Point A to the ELN at Point B (Figs. 1 and 2). Two independent observers performed measurements at different times. The mean of the two measurements was used.

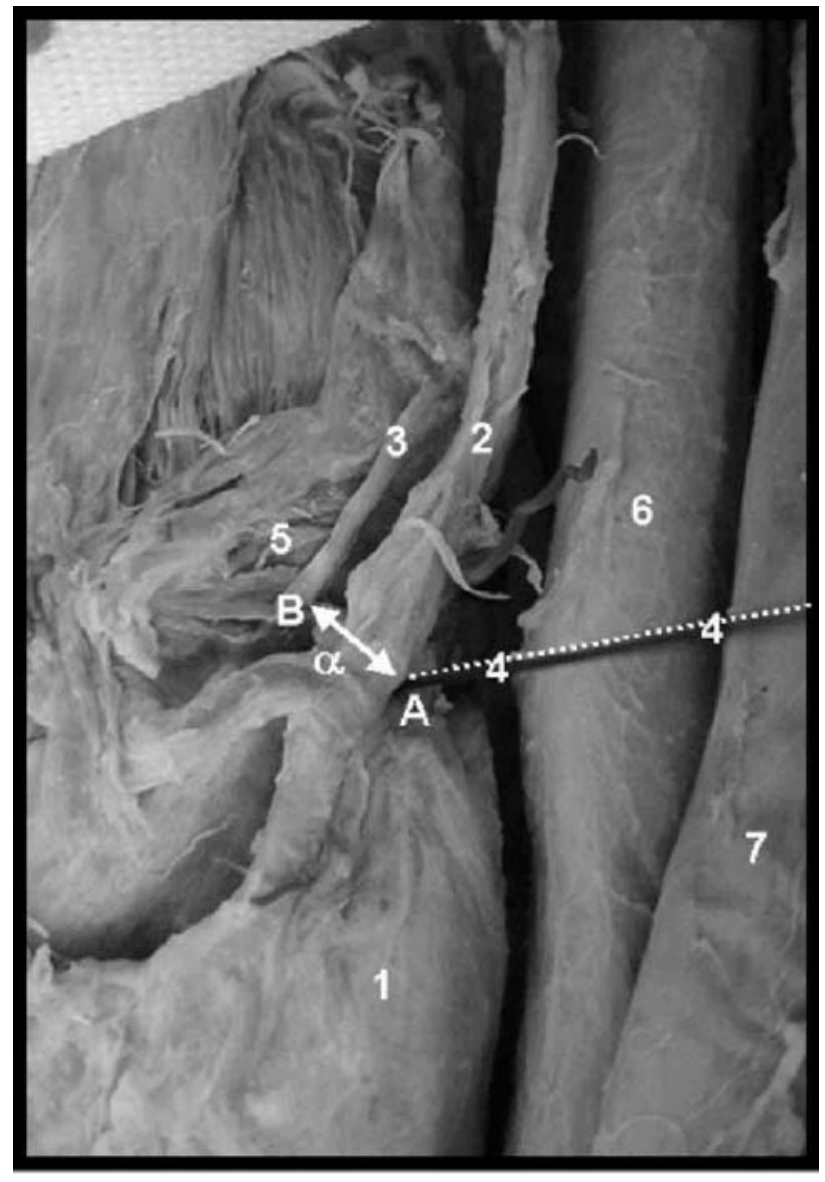

Fig. 1. Dissection of the left lobe of the thyroid gland (1) with the shortest distance a from the superior pole of the thyroid $(A)$ to the external laryngeal nerve (B). 2, STA; 3, ELN; 4, needle inserted on the tip of the superior pole of the thyroid gland; 5 , cricothyroid muscle; 6 , common carotid artery; 7 , internal jugular vein.

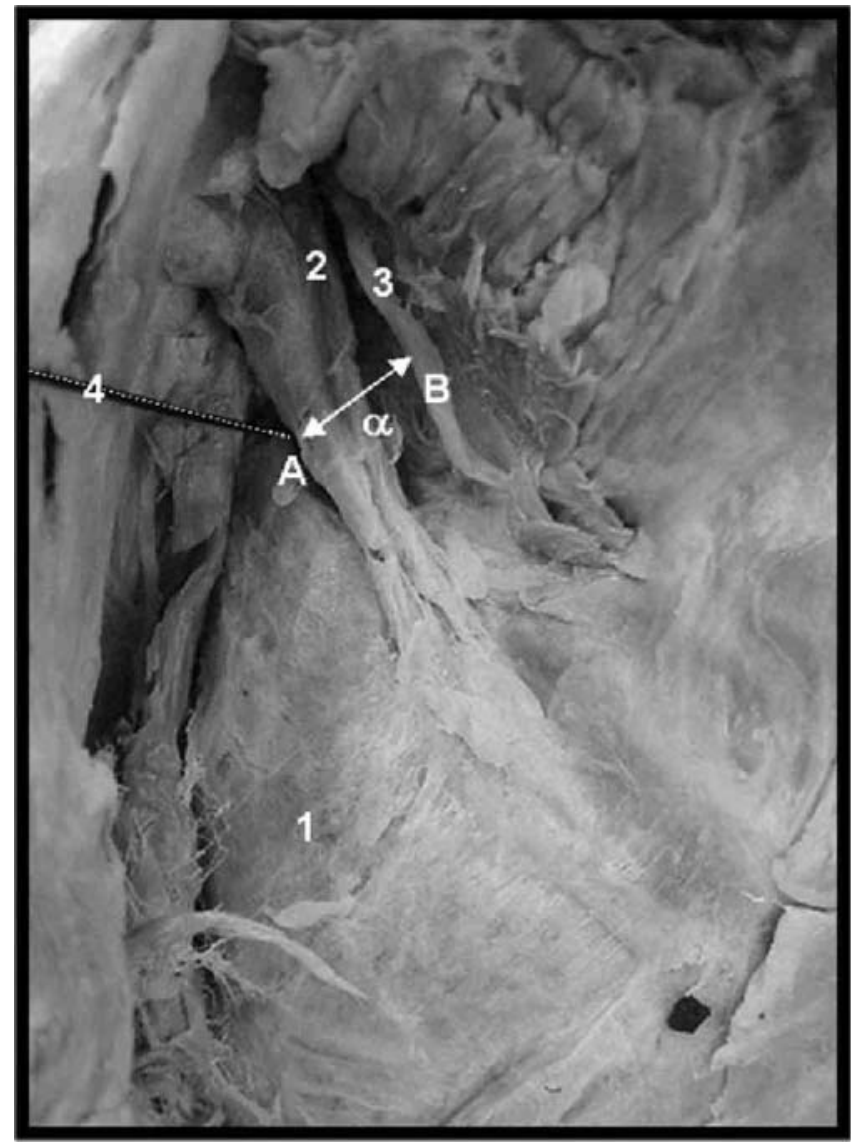

Fig. 2. Dissection of the right lobe of the thyroid gland (1) with the shortest distance a from the superior pole of the thyroid $(A)$ to the external laryngeal nerve (B). 2, STA; 3, ELN; 4, needle inserted on the tip of the superior pole of the thyroid gland. 


\section{RESULTS}

The metric study (Table 1) indicated a mean distance from the most superior point of the superior pole of the thyroid gland (Point A) to the ELN (Point B) of $5.76 \mathrm{~mm}$ on the right, and $6.17 \mathrm{~mm}$ on the left. No ELN was found to run over or inferior to the superior pole of the thyroid gland. Table 2 presents the frequency distribution of the number of cases found in the following categories: $\leq 5$ th percentile $(\leq 2.78 \mathrm{~mm})$; 5 th-25th percentile $(>2.78-\leq 4.46$ $\mathrm{mm})$; 25th-50th percentile (>4.46- $\leq 5.49 \mathrm{~mm})$; 50 th-75th percentile $(>5.49-\leq 6.95 \mathrm{~mm})$; 75th-95th percentile $(>6.95$ $\leq 9.31 \mathrm{~mm})$, and $>95$ th percentile $(>9.31 \mathrm{~mm})$.

The position of the nerve was found to vary considerably with a range of $2.00-11.26 \mathrm{~mm}$ superior to the superior pole of the thyroid gland on the right and $2.78-13.48 \mathrm{~mm}$ on the left.

When using a $90 \%$ coverage of our sample, a range from $2.78 \mathrm{~mm}$ (5th percentile) to $9.31 \mathrm{~mm}$ (95th percentile) from the superior pole of the thyroid gland to the ELN on the right and $3.49 \mathrm{~mm}$ (5th percentile) to $8.66 \mathrm{~mm}$ (95th percentile) on the left was found.

As the lower range of these distances are extremely small on both the left and right, it is imperative to stay on the substance of the thyroid gland, when ligating the superior thyroid vessels during a thyroidectomy. It may even be considered to leave the capsule covering the superior pole of thyroid gland intact when removing the gland to avoid injury to the ELN.

TABLE 1. Statistical Analysis of Results

\begin{tabular}{lcc}
\hline & $\begin{array}{c}\text { Distance } \alpha: \\
\text { (A to B) right }\end{array}$ & $\begin{array}{c}\text { Distance } \alpha: \\
\text { (A to B) left }\end{array}$ \\
\hline Count & 41 & 43 \\
Mean & 5.76 & 6.17 \\
Median & 5.49 & 5.94 \\
Minimum & 2.00 & 2.78 \\
Maximum & 11.26 & 13.48 \\
IR & 0.52 & 0.57 \\
5th percentile & 2.78 & 3.49 \\
25th percentile & 4.46 & 4.80 \\
50th percentile & 5.49 & 5.94 \\
75th percentile & 6.95 & 7.54 \\
95th percentile & 9.31 & 8.66 \\
\hline
\end{tabular}

All measurements are in $\mathrm{mm}$.

TABLE 2. Frequency Distribution: Percentiles of Data Obtained

\begin{tabular}{lclr}
\hline Distance $\alpha$ : (A to B) & Right $(n)$ & Distance $\alpha:($ A to B) & Left $(n)$ \\
\hline 5th percentile $(\leq 2.78)$ & 3 & 55th percentile $(\leq 3.49)$ & 3 \\
5th-25th percentile $(>2.78-\leq 4.46)$ & 9 & 5 th-25th percentile $(>3.49-\leq 4.80)$ & 8 \\
25th-50th percentile $(>4.46-\leq 5.49)$ & 9 & 25th-50th percentile $(>4.80-\leq 5.94)$ & 11 \\
50th-75th percentile $(>5.49-\leq 6.95)$ & 10 & 50th-75th percentile $(>5.94-\leq 7.54)$ & 10 \\
75th-95th percentile $(>6.95-\leq 9.31)$ & 8 & 75th-95th percentile $(>7.54-\leq 8.66)$ & 9 \\
$>95$ th percentile $(>9.31)$ & 2 & $>95$ th percentile $(>8.66)$ & 2 \\
Total & 41 & Total & 43 \\
\hline
\end{tabular}

All measurements in parentheses are in $\mathrm{mm}$. 


\section{DISCUSSION}

The ELN is intimately associated with the STA in close relation to the superior pole of the thyroid gland, thus rendering it vulnerable to injury during ligation of this vessel during thyroid gland surgery (Chosky and Nicholson, 1996; Aina and Hisham, 2001b). It can be stretched by a retractor or transected when the superior thyroid vessels are divided (Chosky and Nicholson, 1996).

Various methods have been described to avoid injury to the ELN. To ensure safety of the ELN, the technique of individual ligation of the superior thyroid vessels close to the medial border of the superior pole, without identifying the nerve was proposed, and even then studies revealed that the risk of injury exceeds 10\% (Lekacos et al., 1987; Cernea et al., 1992a).

Hurtado-Lopez et al. (2005) demonstrated the importance of intraoperative identification of the ELN during thyroidectomies when the showed that the rate of ELN injury increases when not exploring and identifying the nerve expressly. They compared the prevalence of injury during surgery of a 100 superior thyroid poles, divided into three groups; $\mathrm{G} 1$ thyroidectomies, without searching for the ELN, G2 thyroidectomies, searching for the ELN, and G3 a control group. In G1, 25 injuries were found in 15 patients (10 bilaterla and 5 unilateral). In G2, 13 injuries were documented in 8 patients ( 5 bilateral and 3 unilateral). There were no injuries in the control group (G3).

This study revealed that the mean shortest safe distance between the superior pole of the thyroid gland and the ELN on the right side is a mere $5.76 \mathrm{~mm}$, and on the left $6.17 \mathrm{~mm}$. The 5th percentile's confidence interval is even closer to the superior pole of the thyroid gland $(2.78 \mathrm{~mm}$ on the right and $3.49 \mathrm{~mm}$ on the left).

Cernea et al. (1992b) described the classification of the different types of ELNs as follows (Fig. 3):

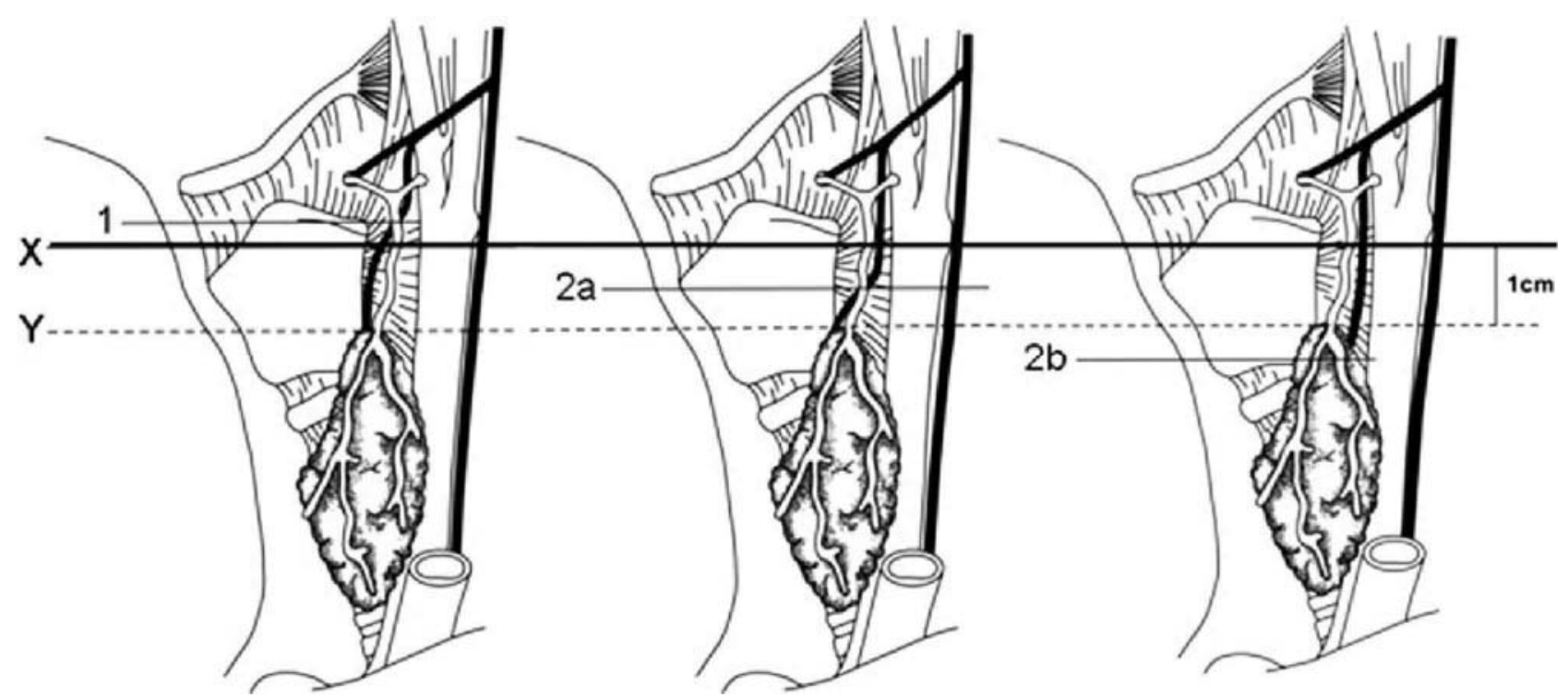

Fig. 3. Schematic representation of the classification of the types of ELNs according to Cernea et al. (1992b), X plane $1 \mathrm{~cm}$ superior to the superior thyroid pole, Y plane at level of the superior thyroid pole. Type 1, ELN crosses the STA superior to X; Type 2a, ELN crosses STA between $X$ and $Y$; Type $2 b$, ELN crosses STA inferior to $Y$. 


\section{openUP}

Type 1: Nerve crossing the superior thyroid vessels 1 or more $\mathrm{cm}$ superior to the apex of the superior thyroid pole, Type 2: Nerve crossing the superior thyroid vessels less than $1 \mathrm{~cm}$ superior or inferior to the apex of the superior thyroid pole, Type 2a: Nerve less than $1 \mathrm{~cm}$ superior the apex of the superior thyroid pole, and Type 2b: Nerve inferior to the apex of the superior thyroid pole.

Type $2 \mathrm{a}$ and $2 \mathrm{~b}$ nerves are considered to be the "nerves at an increased risk" of iatrogenic lesion during a thyroidectomy, owing to their low-lying course (Cernea et al., 1992a,b; Aina and Hisham, 2001b). It is clearly evident from the studies of Cernea et al. that the ELNs are in greater danger in cases of large goiters (type 2: $69 \%$ ), in comparison to those in cases of small goiters (type 2: 25\%) (Cernea et al., 1992a). To support this evidence, Aina and Hisham also found that with large goiters weighing more than $100 \mathrm{~g}$, approximately $92.3 \%$ were type 2 nerves, whereas in goiters weighing less than $100 \mathrm{~g}$, approximately $66.6 \%$ were type 2 nerves (Aina and Hisham, 2001b).

Seven et al. (2003) dissected the thyroid gland and ELN in 30 fresh cadavers (18 females, 12 males; age range 20- 50 years) with a normal thyroid size and no signs of abnormality in the neck, and applied the classification system propesed by Cernea et al. (1992b). They found the ELN was consistent with type 1 in $51.8 \%$ (28 out of 54 lobes) and type 2 in $48.2 \%$ (26 out of 54 lobes); type $2 a$ in $37.1 \%$ and type $2 \mathrm{~b}$ in $11.1 \%$. No significant relationships were found between the type of the nerve, gender, and age.

This suggests that in the case of large goiters in contrast to normal sized thyroid glands, the ELN may be even closer to the superior pole of the thyroid gland and may in fact be crossing over the superior pole. Our study sample excluded pathological conditions, thus our findings apply to "normal thyroid glands." "Normal thyroid glands" are not usually operated on, with the exception of cases where carcinoma is present. On the contrary, other pathological indications such as multi-nodular goiter and Grave's disease, which result in the enlargement of the thyroid gland, do however require thyroid gland surgery. It appears that there is a greater risk of nerve injury in the case of enlarged thyroid glands compared to normal sized thyroid glands.

It is therefore recommended to stay on the superior pole of the thyroid, without going off the gland at all, even leaving a bit of the capsule of the superior pole of the thyroid gland behind. Meticulous dissection and individual ligation of the superior thyroid vessels would be the safest benchmark to avoid injury to the nerve (Aina and Hisham, 2001a). Irrespective of the thyroid pathology encountered, ligation of the superior thyroid vessels should be done as close to the substance of the superior pole of the thyroid gland as possible. Once on the superior pole of the thyroid gland, stay on it making sure that the ELN is not injured by meticulous dissection.

\section{REFERENCES}

Aina EN, Hisham AN. 2001a. External laryngeal nerve in thyroid surgery is the nerve stimulator necessary? Eur J Surg 167:662-665.

Aina EN, Hisham AN. 2001b. External laryngeal nerve in thyroid surgery: recognition and surgical implications. Aust NZ J Surg

71:212-214.

Anonymous. 1970. The recurrent laryngeal nerve at thyroidectomy. Lancet 1:398. 


\section{openUP}

Berry M, Bannister LH, Standring SM. 1995. Nervous system. In: Williams PL, Bannister LH, Berry MM, Collins P, Dyson M, Dussek JE, Ferguson MWJ, editors. Gray's Anatomy. 8th Ed. New York: Churchill Livingston. p 1253.

Cernea CR, Ferraz AR, Furlani J, Monteiro S, Nishio S, Hojaij FC, Dutra Junior A, Marques LA, Pontes PA, Bevilacqua RG. 1992a. Identification of the external branch of the superior laryngeal nerve during thyroidectomy. Am J Surg 164:634- 639.

Cernea CR, Ferraz AR, Nishio S, Dutra A Jr, Hojaij FC, dos Santos LR. 1992b. Surgical anatomy of the external branch of the superior laryngeal nerve. Head Neck 14:380-383.

Choksy SA, Nicholson ML. 1996. Prevention of voice change in singers undergoing thyroidectomy by using a nerve stimulator to identify the external laryngeal nerve. Br J Surg 83:1131- 1132.

Delbridge L, Reeve TS, Khadra M, Poole AG. 1992. Total thyroidectomy: The technique of capsular dissection. Aust N Z J Surg 62:96-99.

Droulias C, Tzinas S, Harlaftis N, Akin JT Jr, Gray SW, Skandalakis JE. 1976. The superior laryngeal nerve. Am Surg 42:635638.

Durham CF, Harrison TS. 1964. The surgical anatomy of the superior laryngeal nerve. Surg Gynecol Obstet 118:38-44.

Friedman M, Toriumi DM. 1986. Functional identification of the external laryngeal nerve during thyroidectomy. Laryngoscope 96:1291-1292.

Hurtado-Lopez LM, Pacheco-Alvarez MI, Montes-Castillo Mde L, Zaldivar-Ramirez FR. 2005. Importance of the intraoperative identification of the external branch of the superior laryngeal nerve during thyroidectomy: Electromyographic evaluation. Thyroid 15:449-454.

Lekacos NL, Miligos ND, Tzardis PJ, Majiatis S, Patoulis J. 1987. The superior laryngeal nerve in thyroidectomy. Am Surg 53:610- 612.

Lennquist S, Cahlin C, Smeds S. 1987. The superior laryngeal nerve in thyroid surgery. Surgery 102:999-1008.

Moosman DA, DeWeese MS. 1968. The external laryngeal nerve as related to thyroidectomy. Surg Gynecol Obstet 127:10111016.

Moreau S, Goullet de Rugy M, Babin E, Salame E, Delmas P, Valdazo A. 1998. The recurrent laryngeal nerve: Related vascular anatomy. Laryngoscope 108:1351-1353.

Seven H, Alkan Z, Cakir BO, Sam B, Uslu B, Turgut S. 2003. The topographical anatomy of the external branch of the superior laryngeal nerve and its importance in thyroid surgery: A cadaver study. Kulak Burun Bogaz Ihtis Derg 11: 161-165.

Teitelbaum BJ, Wenig BL. 1995. Superior laryngeal nerve injury from thyroid surgery. Head Neck 17:36-40.

Wagner HE, Seiler C. 1994. Recurrent laryngeal nerve palsy after thyroid gland surgery. Br J Surg 81:226-228. 